\title{
A PRACTICAL MODEL FOR ENDODONTIC RADIOGRAPHY TEACHING
}

Z.A. Che Abd. Aziz. A practical model for endodontic radiographic teaching. Annal Dent Univ Malaya 2008; 15(2): 67-70.

\section{ABSTRACT}

Aim: To manufacture a clinical simulation apparatus for the undergraduates'endodontic radiography teaching

Objectives:

- To provide a model for teaching of parallax method using Kelly's forcep

- To provide a model for undergraduates to practice radiographic localization employing parallax method.

- To allow students to practice taking radiographs in a way that simulates the clinical situations with a good diagnostic quality

Methods: Impressions of a dentate arch (maxillary and mandibullary) were used to form a stone cast. A section of the cast, in the area where the natural teeth were to be placed, is sectioned and removed. Three maxillary extracted teeth (canine, first and second premolar) were selected and mounted with acrylic resin at the sectioned area. The resin was cured in a light box. The arches were mounted in a phantom head with a placement of rubber cheek. The first premolar was isolated with rubber dam. The intraoral holder (Kelly's forcep) was attached to a robotic arm. The students were taught the correct angulations of the $\mathrm{x}$-ray cone for the paralleling technique and parallax method using Kelly's forcep during root canal treatment.

Results: All students managed to complete the exercise and were considered competent when they produced acceptable quality of radiographs.

Conclusion: The model described was improvised from a model that has been used during the past 2 years for undergraduates' endodontic courses. It has been well accepted as it simulates the clinical situation more closely than was possible previously.

Key words: simulation apparatus; endodontic radiography teaching

\section{INTRODUCTION}

Radiographs taken during the treatment phase of root canal therapy, are used to determine working lengths and to check the adequacy of obturation. The importance of obtaining an acceptable quality of root canal treatment films was emphasized by
Original Article

\author{
Z.A. Che Abd. Aziz \\ Department of Conservative Dentistry \\ Faculty of Dentistry \\ University of Malaya \\ 50603 Kuala Lumpur \\ Malaysia
}

Corresponding author: Zeti Adura Che Abd. Aziz

Swartz (1), who stated that the shopistication of root canal treatment (RCT) closely parallels the sophistication of RCT radiographic techniques. When the films are inaccurate and have to be retaken, RCT becomes less efficient, and patients are exposed to additional radiation. Torabinejad et al. (2) reported that $\mathrm{x}$-rays doses varied among patients on whom root canal treatment films were taken, but these doses were relatively low. However, risk does occur, therefore they recommended that the number of radiographs taken to be kept to a minimum.

A study conducted by Bean in 1969 reported that an $11 \%$ incidence of radiographic errors when film were taken by junior dental students with the use of Rinn's XCP instrument (Rinn Corp., Elgin, I11.) with the paralleling technique. The incidence increased to $25 \%$ for junior and senior students who used the bisecting angle technique (BAT) and handheld films. The authors attributed the higher success rate of the first group to the use of paralleling technique and film holders. The rate of radiographic errors that occur when endodontic treatment films (ETFs) are taken has not been determined. The studies published mainly concerned with distortion. Lim and Teo (3) compared the amount of distortion or magnification that occurred on preoperative nonendodontic films to that ETFs taken on the single-rooted anterior and premolar teeth. They found that more distortion occurred with ETFs than with nonendodontic films with maxillary premolars having the highest incidence of distortion. Interestingly, Forsberg $(4,5)$ measured these discrepancies in the working length. ETFs taken with bisecting angle technique and handheld films were compared with ETFs taken using paralleling technique and film holders. $\mathrm{He}$ found that $20 \%$ of ETFs taken with bisecting angle technique had distortion over $1 \mathrm{~mm}$, where the endodontic files were uner- or over-extended. However, under similar conditions only $4 \%$ of the ETFs taken with paralleling technique had over $1 \mathrm{~mm}$ distortion. 
With respect to film holders, there are devices or special adaptations of paralleling devices that can be used for endodontic working films such as XCP instruments, Rinn's Snap A Ray II (Rinn. Corp.), Unibite (Identoflex Co., Switzerland) and EndoRay (Rinn. Corp.). There have been designed where the patient can bite on it or the presence of positioning arm provides for $\mathrm{x}$-ray cone alignment or both (6). However, with some practice, nothing is more effective than a hemostat (Kelly's forcep) for its adaptability in the oral cavity. Furthermore the hemostat can also be placed and sterilized in a kit with other dental instruments. As you probably aware, patients are discouraged to hold the films with direct finger pressure. This frequently results in bent films with distorted radiographic images. In addition, besides being cheaper than other film holders it is also readily available in a dental clinic. Nevertheless for a first-time user, he requires guidance for proper $\mathrm{x}$-ray cone alignmen, especially with the presence of rubber dam. Thus it is important for the students to practise the taking of ETFs on a practical model prior to embarking to treat patients on patients (8). In this paper we describe the practical model that was designed to allow good quality radiographs to be taken during root canal treatment using the Kelly's forcep and robotic arm.

\section{MATERIALS AND METHODS}

Impressions of dentate arches (maxillary and mandibular) were used to form stone casts, in a model tray designed for use in crown and bridge construction. A section of the maxillary cast, in the area where natural teeth are to be placed, is sectioned and removed. Three extracted teeth (canine, first and second maxillary premolars) were selected and cleaned. The teeth were mounted in light-curing acrylic resin (Megatray light-curing resin, Megadenta GMbH, Germany) at the sectioned area. The resin was then moulded by hand to reproduce the soft tissue contour, to ensure the teeth are secured at the cervical margin (Fig. 1). The maxillary cast is then placed in the light box to cure the resin. Both the casts are then mounted in a phantom head. Rubber cheek is then placed. The first premolar is then isolated with a rubber dam (Fig. 2). A robotic arm which simulates a patientholding-the hemostat is used to hold the forcep. These apparatus (phantom head and robotic arm) are mounted on a wheel steel rod stand (Fig. 3).

The students were then taught the correct alignment and angulation of the $\mathrm{x}$-ray cone for the paralleling technique. The students were then instructed to produce an acceptable quality radiograph of the first maxillary premolar employing the parallax method. This technique enables the buccal and palatal roots to become separated (Fig.

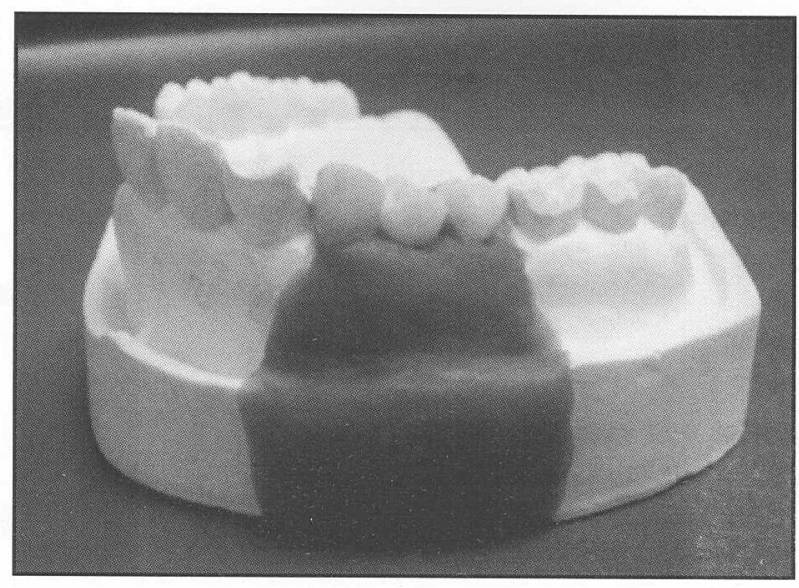

Figure 1: Extracted teeth embedded in resin were mounted in the maxillary plaster arch.

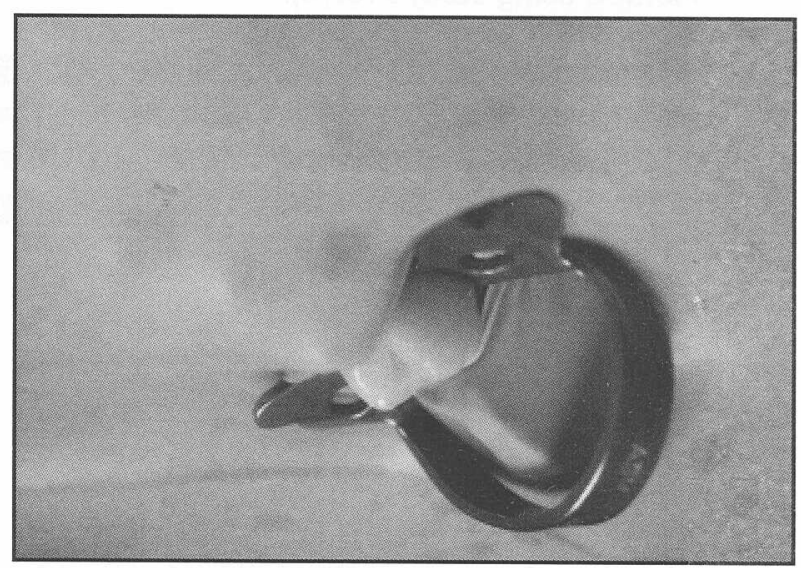

Figure 2: Single-tooth isolation with rubber dam of first maxillary premolar.

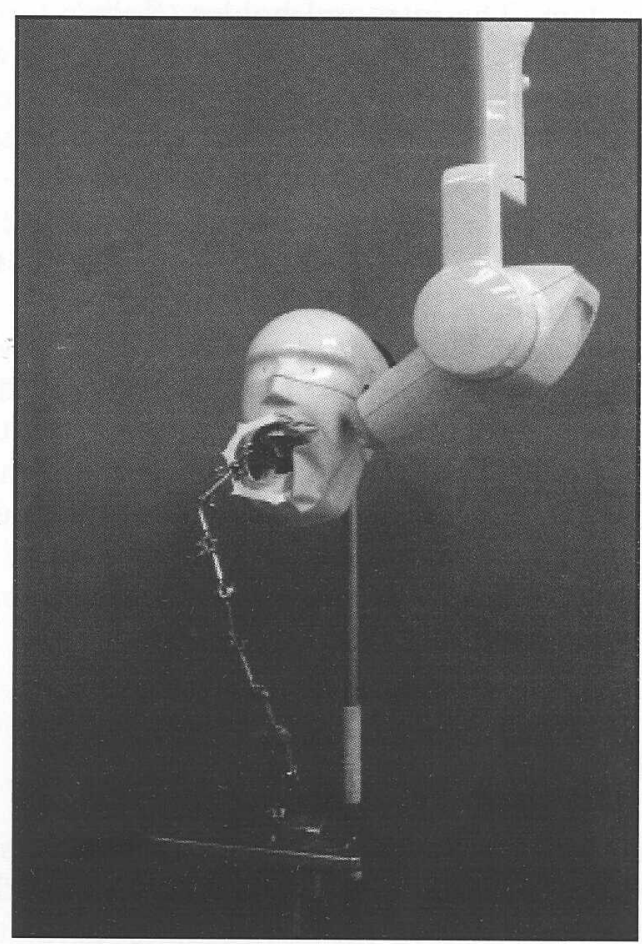

Figure 3: Phantom head and robotic arm mounted on a rod stand. 
4). The film was at $90^{\circ}$ angle with the handle of Kelly's forcep when been clipped on it (Fig. 5). The hemostat-held film is then placed in the oral cavity and adjusted so that the film approximately parallel to the first premolar axial plane. The cone was then aligned parallel to the hemostat in the frontal plane (vertical angulation) (Fig. 6) and at $90^{\circ}$ to the handles (Fig. 6). The radiographic beams would then be striking the film at the $90^{\circ}$ angle.

\section{DISCUSSION}

A durable model can be constructed from light curing acrylic resin in a preformed tray. The resin is unaffected by water from the high-speed handpiece. Based on our experience the presence of water caused deterioration of models constructed from sawdust and plaster even though covered with heated wax. The use of light-curing acrylic resin as a mounted medium at the sectioned maxillary cast has greatly improved the clarity of the image on the radiograph produced. This is probably attributed to the less radiodense mounting medium compared to if the teeth were mounted directly onto plaster of Paris. Prior to the use of light-cure acrylic resin, various portions of acrylic resin powder has been mixed with plaster of Paris. However the image produced was not as clear as using the light-curing resin as done in this study.

As the casts were mounted onto a phantom head and a rubber dam could be applied, this model can also be used to practise endodontic pre-clinical exercises. Concurrently, exercises for endodontic radiography could be done either using hemostat as the film-holder or paralleling device (EndoRay, Rinn Corporation, Illionis, USA). This pre-clinical endodontic teaching model is certainly simulating closely the clinical situation. A reliable radiographic technique is important in endodontics as inadequate visualization of apices may affect the outcome of treatment (4).

To ensure the students grasp the practical skills of endodontic radiographic taking instead only the theoretical aspect, they were required to produce a good preoperative radiograph (on maxillary first premolar) with parallax view. This is important as study shown that many clinician had difficulty to produce an acceptable parallax view radiograph of maxillary first premolar $(4,5)$. This was mainly due to the anatomical factor (6). Inability to do so will cause inaccuracy during diagnosis stage. This furthermore would reduce the risk of disturbing the moisture control property of rubber dam as removing the rubber dam's frame during radiograph taking would decrease its efficiency especially at the gingival margins (7).

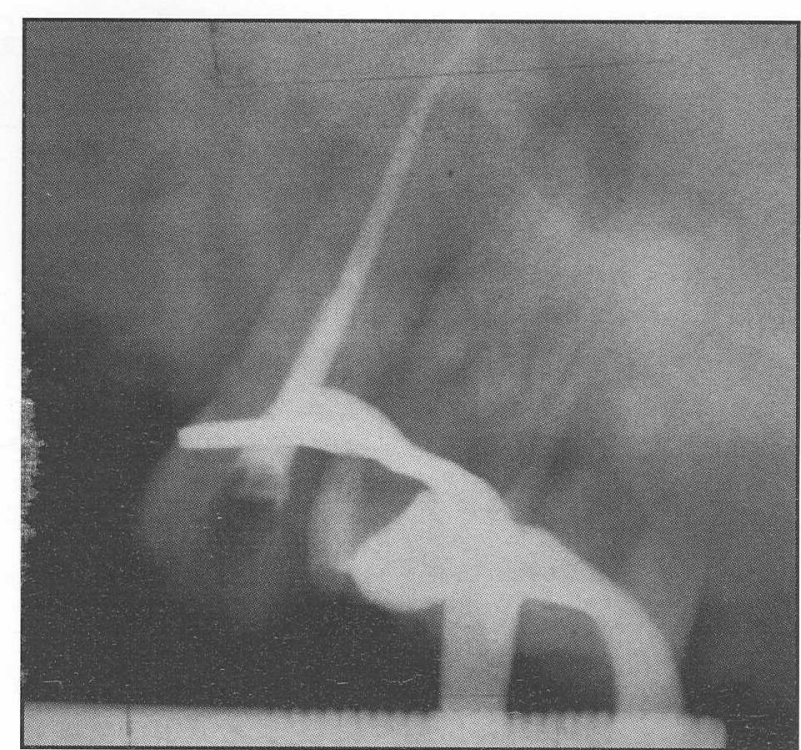

Figure 4: Separation images of buccal and palatal roots of first maxillary premolar.

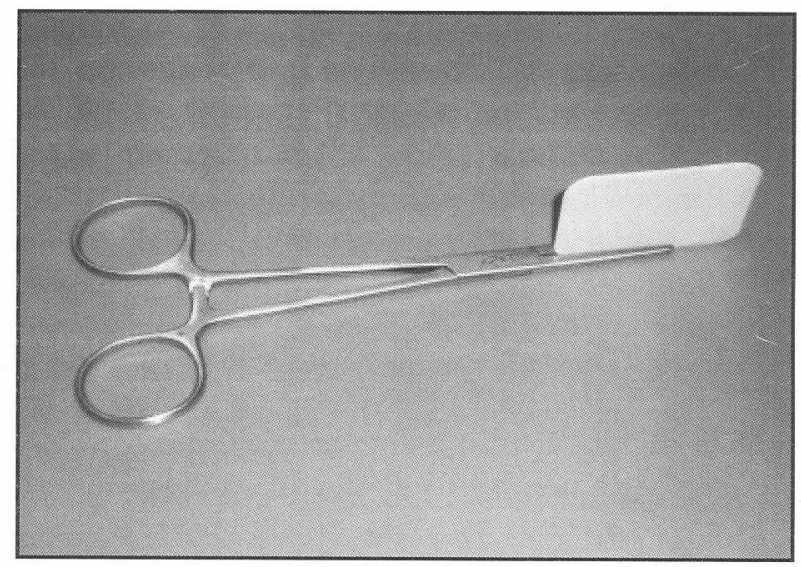

Figure 5: Periapical film at the $90^{\circ}$ with the Kelly's forcep handle.

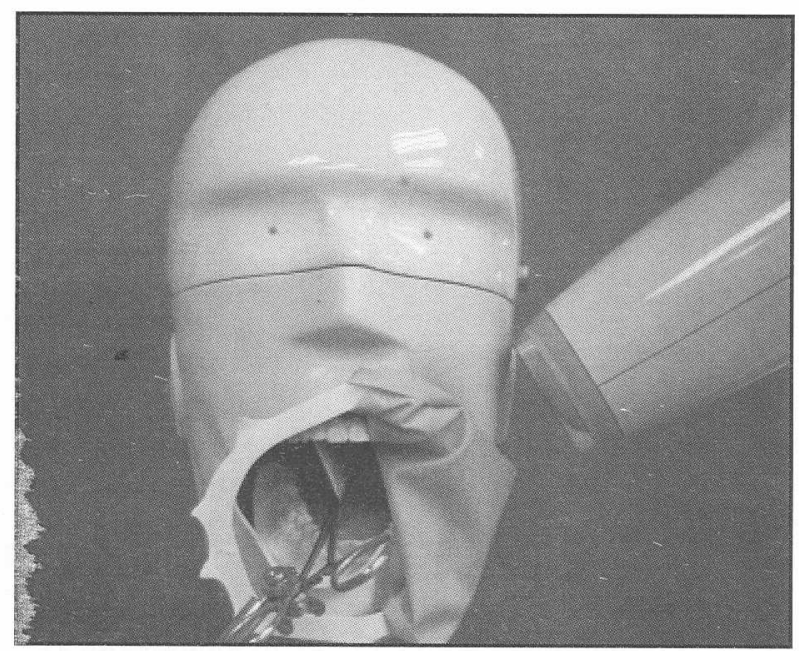

Figure 6: The periapical film is at $90^{\circ}$ with the radiograph cone. 


\section{CONCLUSION}

The model described was improvised from a model that had been used for the past 2 years for undergraduate radiographic teaching during root canal treatment. It has been well accepted by the students as they have the chance to practise the parallel view technique without the worry of overexposure of radiation imposed on the patient. Furthermore it would make their clinical skill and time more efficient.

\section{ACKNOWLEDGEMENTS}

The author would like to thank Mr. Haripin, Dental Assistant at Operative Laboratory for his assistance with this project.

\section{REFERENCES}

1. Schwartz S. Preparation for therapy. In: Cohen S, Burns R, eds. Pathways to the pulp, $2^{\text {nd }}$ ed. St. Louis: CV Mosby, 1980: 47.

2. Torabinejad M, Danforth R, Andrews K, Chan C. Absorbed radiation by various tissues during simulated endodontic radiography. J Endod 1989; $15: 249-53$.
3. Lim K, Teo C. Some problems encountered in endodontic radiography. Ann Acad Med Singapore 1986; 15: 320-5.

4. Forsberg J. A comparison of the paralleling and bisecting angle radiographic techniques in endodontics. Int Endo J 1987; 20: 177-82.

5. Forsberg J. Estimation of the root filling length with the paralleling and bisecting angle techniques performed by undergraduates' students. Int Endo J 1987; 20: 282-6.

6. Gound G, DuBois L, Biggs S. Factors that effect the rate of retakes for endodontic treatment radiographs. Oral Surg Oral Med Oral Pathol 1994; 77: 514-8.

7. Sauveur G. Improvement of the rubber dam frame. J Endod 1997; 23(12): 765-7.

8. Nattress BR, Manogue M, Carmichael. A model for the teaching of clinical techniques in root canal treatment. Int. Endod. J 1997; 30: 343-6. 